\title{
The Effect of Particle Size on the Optical and Electronic Properties of Magnesium Oxide Nanoparticles
}

\author{
Martijn A. Zwijnenburg \\ Department of Chemistry, University College London, 20 Gordon Street, \\ London WC1H 0AJ, U.K. \\ Email: m.zwijnenburg@ucl.ac.uk
}

The quasiparticle states, fundamental gaps, optical gaps, exciton binding energies and UV-Vis spectra for a series of cuboidal nanoparticles of the prototypical oxide magnesium oxide $(\mathrm{MgO})$, the largest of which has 216 atoms and edges of $1 \mathrm{~nm}$, were predicted using many-body perturbation theory (evGW-BSE). The evolution of the properties with particle size was explicitly studied. It was found that while the highest occupied and lowest unoccupied quasiparticle states and fundamental gap change with particle size, the optical gap remains essentially fixed for all but the smallest nanoparticles, in line with what was previously observed experimentally. The explanation for these observations is demonstrated to be that while the optical gap is associated with an exciton that is highly localised around the particle's corner atoms, the highest occupied and lowest unoccupied quasiparticle states, while primarily localised on the oxygen corner atoms (hole) and magnesium corner atoms (electron), show significant delocalisation along the edges. The strong localisation of the exciton associated with the optical gap on the corner atoms is argued to also explain why the nanoparticles have a much smaller optical gaps and red-shifted spectra than bulk $\mathrm{MgO}$. Finally, it is discussed how this non-quantum confinement behaviour, where the properties of the nanoparticles arise from surface defects rather than differences in localisation of edge or exciton states, appears typical of alkaline earth oxide nanoparticles, and that the true optical gap of bulk crystals of such materials is also probably the result of surface defects, even if unobservable experimentally.

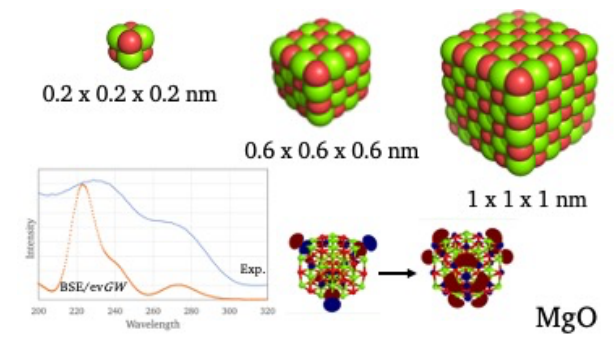




\section{Introduction}

The effect of going from the bulk to nanosized particles on the optical and electronic properties of materials is interesting both from a fundamental and applied perspective. ${ }^{1}$ Practically it offers an alternative way of tuning material properties besides changing composition while conceptually it raises questions about what exactly results in the properties of nanoparticles differing from those of the bulk. Quantum confinement, ${ }^{2-3}$ where the size of the particle constrains the size of the exciton --the excited-electron hole pair formed by the absorption of light-- to a size smaller than in the bulk, is often invoked but requires the states involved to be delocalised over the particle, which is unlikely to be the case for more ionic materials. Quantum confinement also only explains blue-shifts with respect to the bulk, as e.g. observed for $\mathrm{CdS},{ }^{4} \mathrm{CdSe},{ }^{5-6} \mathrm{PbS}^{7-8}$ and $\mathrm{PBSe}^{9}$ nanoparticles, but not red-shifts, as e.g. observed for $\mathrm{MgO},{ }^{10-11} \mathrm{CaO}^{11}$ and $\mathrm{SrO}^{12}$ particles. An alternative mechanism by which the properties of nanoparticles may differ from the bulk involves the localisation of relevant states on low-coordinated surface atoms, which are ubiquitous on (small) nanoparticles. While experimental spectroscopy can clearly demonstrate the effect of nanostructuring on the electronic and optical properties, elucidating the atomic scale origin of these changes requires the combination of experiment with theory.

Magnesium oxide (MgO) nanoparticles are an ideal system to study differences in the optical and electronic properties between nanoparticles and the bulk and to synthesize the results of experiment and theory. Well-defined $\mathrm{MgO}$ nanoparticles as small as $3 \mathrm{~nm}$ have been prepared experimentally by means of chemical vapour deposition without the need for capping agents and found to display UV-Vis diffuse reflection and photoluminescence spectra that are significantly red-shifted relative to bulk magnesium oxide. ${ }^{10-11}$ The lowest-energy most red-shifted exciton peak in the reflection spectrum of bulk $\mathrm{MgO},{ }^{13-14}$ the bulk optical gap, is located at $7.7 \mathrm{eV}(161 \mathrm{~nm})$ while the lowest peak in the reflection spectrum of the $3 \mathrm{~nm}$ particles lies at $\sim 4.6 \mathrm{eV}$ $(270 \mathrm{~nm}) .{ }^{10}$ These $3 \mathrm{~nm}$ nanoparticles are confirmed to have the same rocksalt structure as bulk magnesium oxide, ruling out that the observed red shift is the result of major structural changes. Indeed, computational global optimisation studies show that in contrast to materials that in the bulk crystallise with the zincblende or wurtzite 
structure, e.g. zinc or cadmium sulfide ${ }^{15-19}$ the lowest energy nanoparticles of materials that crystallise with the rocksalt structure, such as $\mathrm{MgO}^{20-22}$ are, even in the absence of capping agents, generally cuts from that crystal structure. The fact that magnesium and oxygen are relatively light elements also means that relativistic effects including spinorbit coupling will be small in $\mathrm{MgO}$ nanoparticles in contrast to other rocksalt nanoparticles, such as those made from $\mathrm{PbS}$ or $\mathrm{PbSe}$. Finally, while the $\mathrm{MgO}$ nanoparticles' UV-Vis spectra are significantly red-shifted with respect to the bulk, the largest change in the diffuse reflectance spectra when changing the average particle size from 3 to $10 \mathrm{~nm}$ is not a shift in the peak positions but their relative intensities. ${ }^{10}$ Something that has been proposed based on quantum chemistry calculations on computational models of corners, edges and faces of $\mathrm{MgO}$ nanoparticles, ${ }^{23-26}$ see below, to result from the fact that the lowest energy excited states in such particles localises on the corners and edges rather than being delocalised over the volume of the particle. A proposal that we here will re-investigate by calculations on whole nanoparticles.

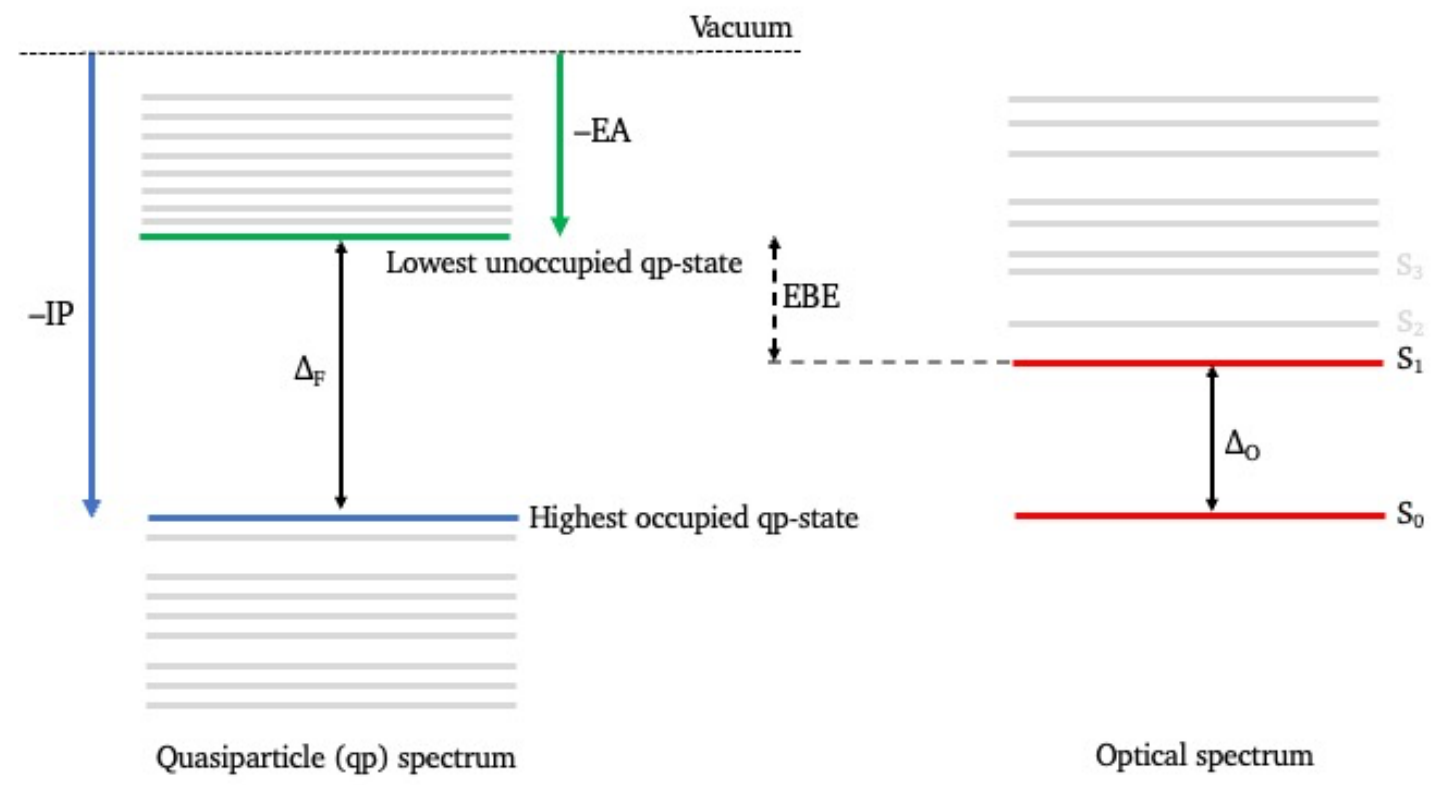

Fig. 1 Cartoon illustrating the definition of the highest occupied and lowest unoccupied quasiparticle states (-IP and -EA), the fundamental gap $\left(\Delta_{\mathrm{F}}\right)$, optical gap $\left(\Delta_{\mathrm{O}}\right)$, and the exciton binding energy (EBE).

In contrast to the case of the optical properties, there appear to be no experimental reports on the electronic structure of $\mathrm{MgO}$ nanoparticles: i.e. the 
fundamental gap, the energy required to generate a non-interacting excited electron and hole pair rather than an interacting exciton and/or the energies of the highest occupied (the negative of the ionisation potential, -IP) and lowest unoccupied (the negative of the electron affinity, -EA) quasiparticle states (see Fig. 1). The latter concepts in the case of periodic crystals, where it is appropriate to speak of bands, map on to the bandgap, valence band maximum and conduction band minimum, respectively. There are some reports in the literature of the fundamental gap narrowing for thin films of $\mathrm{MgO}^{27-28}$ However, as this is generally observed by means of electron energy loss spectroscopy (EELS) and as one of the loss mechanisms in EELS involves the generation of excitons, the feature at $\sim 6 \mathrm{eV}$ in the EELS spectrum of thin magnesium oxide films linked to the apparent narrowing of the fundamental gap in reality might be due to surface exciton formation ${ }^{29-30}$ and thus be evidence of the narrowing of the thin films' optical gap instead.

The optical properties of $\mathrm{MgO}$ nanoparticles were previously studied ${ }^{23-26,31}$ using time-dependent density functional theory (TD-DFT). The results of these TD-DFT calculations for whole nanoparticles were found to be rather sensitive to the exact density functional used ${ }^{31}$ because of the well-known issue ${ }^{32-33}$ of TD-DFT where chargetransfer excitations are spuriously stabilised with respect to local, i.e. non-chargetransfer, excitations. Well-chosen density functionals with the optimal amount of exact exchange can reproduce the key features of the experimental spectra of magnesium oxide nanoparticles: the red shift with respect to the bulk and the intensity change for the diffuse reflection peaks when changing particle size,${ }^{31}$ but the strong dependency on the amount of exact exchange makes these calculations more empirical than desirable. TD-DFT calculations using embedded cluster calculations ${ }^{23-26}$ where only a region, e.g. a corner, of the nanoparticle is describe explicitly using TD-DFT and the effect of the rest of the particle on this region is described in terms of classical point charges, appear to be less sensitive to the functional choice, perhaps because many potential charge-transfer excitations, e.g. from oxygen corner atoms to magnesium corner atoms, are by definition absent in embedded cluster models. ${ }^{31}$ It is however difficult to explicitly study the effect of particle size in embedded cluster calculations. An additional complication when using TD-DFT is that the optical gap and the UV-Vis 
spectra in general are not treated on the same footing as the particle's electronic properties. The highest occupied and lowest unoccupied quasiparticle states of a particle and its fundamental gap can in principle be calculated within the framework of ground state density functional theory (DFT) in two different ways, from the KohnSham (KS) orbital energies or using $\triangle \mathrm{DFT}$. In the former case, the highest occupied and lowest uncopied quasiparticle states map on to the highest occupied and lowest unoccupied KS orbitals and the fundamental gap on to the energy gap between these two orbitals, the KS gap. In the latter case the energy of the highest occupied quasiparticle state is calculated from the difference in total energy between the neutral particle and the particle with one electron less, that of the lowest unoccupied quasiparticle state from the total energy difference between the neutral particle and the particle with an extra electron, and the fundamental gap from the difference in energy between the lowest unoccupied and highest occupied quasiparticle states. The $\triangle \mathrm{DFT}$ approach is preferred as it can be shown that the KS gap, at least for pure density functionals, behaves more like the optical than the fundamental gap because the KS unoccupied orbitals feel the same field of N-1 electrons as the KS occupied orbitals instead of the correct $\mathrm{N}$ electrons. ${ }^{34}$ However, regardless, it is not a given that the optimal density functional for calculating the spectra and optical gap values of particles is also optimal for calculating the particles' electronic properties. This is especially critical for properties such as the exciton binding energy, the difference between the fundamental and optical gap and a measure of how strongly excitons are bound (see Fig. 1), which span both worlds. $\Delta$ DFT predictions of the highest occupied and lowest unoccupied quasiparticle energies of $\mathrm{MgO}$ particles, including particles with defects or in the presence of grain boundaries, have previously been reported by Shluger and coworkers based on embedded cluster calculations. ${ }^{35-37}$ 
A

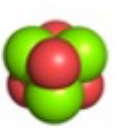

B

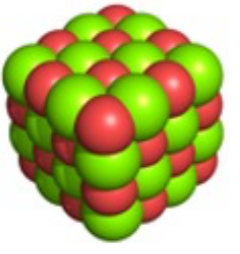

$\mathrm{C}$

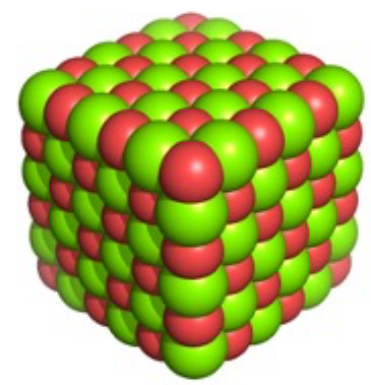

$\mathrm{D}$

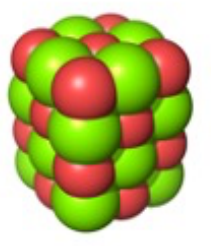

E

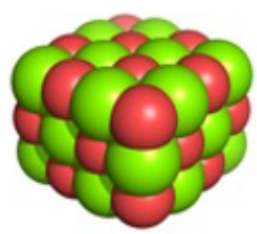

$\mathrm{F}$

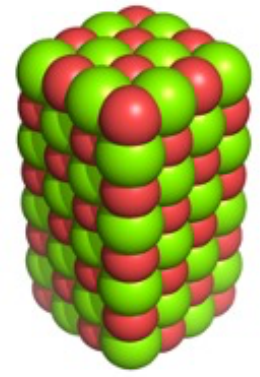

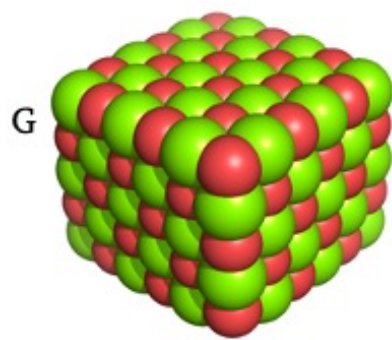

Fig. 2 Structures of some of the cuboidal particles studied. The cubes $(\mathrm{MgO})_{4}(\mathrm{~A})$, $(\mathrm{MgO})_{32}(\mathrm{~B})$ and $(\mathrm{MgO})_{108}(\mathrm{C})$, as well as the cuboids $(\mathrm{MgO})_{18}(\mathrm{D}),(\mathrm{MgO})_{24}(\mathrm{E})$, $(\mathrm{MgO})_{64}(\mathrm{~F})$ and $(\mathrm{MgO})_{90}(\mathrm{G})$.

Here many-body perturbation theory, in the form of solving Hedin's GW equations $^{38-40}$ followed by the Bethe-Salpeter equation, ${ }^{41-43}$ is used to calculate the optical and electronic properties of cuboidal $\mathrm{MgO}$ rocksalt nanoparticles, see Fig. 2, rather than (TD-)DFT. While computationally considerably more expensive than (TD)DFT, GW-BSE has the advantage that it treats optical and electronic properties on a similar footing and that application of partial self-consistency in the $G W$ part of the calculation, evGW, removes most of the dependency on the specific density functional used in the underlying DFT calculation. Using GW-BSE the effect of particle size on the optical and fundamental gap of $\mathrm{MgO}$ nanoparticles, the localisation of the quasiparticle and excited state, as well as the excitonic character of the latter, is explored in order to explain the atomistic scale explanation of the differences between the nanoparticle properties and those of the bulk. By performing calculations on whole nanoparticles rather than embedded cluster calculations we can explore the effect of particle-size directly, as well as the interplay between different sites on which the excited state or quasiparticles can localise. The results of our calculations are not only relevant to understand the properties of $\mathrm{MgO}$ nanoparticles but also lie the groundwork for future work on (rocksalt) nanoparticles of other materials. 


\section{Methodology}

The geometry of the nanoparticles was optimised in DFT calculations, using the B3LYP $^{44-47}$ density functional in combination with the D3(BJ) ${ }^{48-49}$ dispersion correction by Grimme and co-workers and either the def2-SVP or def2-TZVPP basis-set. ${ }^{50}$ During the geometry optimisation the symmetry of the nanoparticles was fixed and for the smaller particles, up to and including $(\mathrm{MgO})_{32}$, frequency calculations were performed to verify that the optimised structures correspond to minima.

Single-shot $G_{0} W_{0}$, eigenvalue-only self-consistent $G W(\mathrm{evGW})$ and quasiparticle self-consistent (qsGW) calculations, as implemented by Holzer, van Setten, Klopper and co-workers in Turbomole, ${ }^{51-53}$ were performed on the DFT optimised structures, starting from B3LYP orbitals and in selected case $\mathrm{PBE}^{54}$ orbitals. These calculations used either the def2-SVP or def2-TZVPP basis-set (and in selected cases the def2-QZVPP and aug-cc-pVTZ basis-sets) and one of two $G W$ implementations that differ in how the selfenergy is obtained; in terms of a spectral representation (SR) or by analytical continuation (AC). The $G W$ calculations using the spectral representation are the most computationally expensive, scaling as $\mathrm{N}^{6}$, while the analytical continuation calculations are more computationally tractable, scaling as $\mathrm{N}^{4}$. In the case of analytical continuation only the two edge-states are explicitly calculated with $G W$ and the rest of the orbitals simply shifted. The results of the different $G W$ calculations are used as input for solving the BSE, again as implemented in Turbomole, ${ }^{55}$ to obtain excitation energies and oscillator strength values. The character of the BSE excited-states is finally analysed in terms of the most prominent natural transition orbitals. ${ }^{56}$ The same basis-sets are generally used for the geometry optimisation and the BSE-GW calculations, except where stated to the contrary.

For selected particles the lowest excitation energies are also calculated with LRCCSD coupled-cluster theory on top of a Hartree-Fock ground-state. These correlated wavefunction calculations use either the def2-TZVPP or def2-QZVPP basis-set and are performed as single-point calculations on the DFT optimised geometry.

All calculations were performed using version 7.5 of the Turbomole code ${ }^{57}$ and used a tight integration grid (m5) and tight SCF convergence criteria (scftol and denconv $1 \times 10^{-7}$ ). All GW and BSE calculations additionally used the RI-K approximation 
and use of symmetry was limited to Abelian point groups. The $G W$ and BSE calculations for $(\mathrm{MgO})_{4},(\mathrm{MgO})_{32}$ and $(\mathrm{MgO})_{108}$, as well as $(\mathrm{CaO})_{32}$ and $(\mathrm{SrO})_{32}$, all of which have $T$ d symmetry, were hence performed in the $D_{2}$ point group instead.

\section{Results}

$(\mathrm{MgO})_{4}$ and $(\mathrm{MgO})_{32}$.

First, the effect of the different $G W$ approximations and implementations is studied by performing calculations on the smallest two perfect cubes, $(\mathrm{MgO})_{4}$ and $(\mathrm{MgO})_{32}$. Tables 1 and 2 give the highest occupied and lowest unoccupied quasiparticle energies, fundamental gap, optical gap and exciton binding energy values predicted by the different method combinations for the two particles. Concentrating first on the effect of basis-set, with increasing basis-set size the highest occupied and lowest unoccupied quasiparticle states both move to deeper, more negative, values while the fundamental and optical gap increase and the exciton binding energy more or less remains the same. Generally, as would be expected, the shift when going from the triple-zeta def2-TZVPP to the quadruple-zeta def2-QZVPP basis-set is smaller than when going from def2-SVP to def2-TZVPP, except for the lowest unoccupied quasiparticle state, for which the shift is similar. A calculation using the augmented Dunning aug-cc-pVTZ basis-set, suggest that the effect of adding additional diffuse basis functions is minor.

Table 1 Highest occupied (-IP) and lowest unoccupied (-EA) quasiparticle states, fundamental gap $\left(\Delta_{\mathrm{F}}\right)$, optical gap $\left(\Delta_{\mathrm{O}}\right)$ and exciton binding energy (EBE) values of $(\mathrm{MgO})_{4}$ as calculated by the different method combinations (TZVPP $=$ def2-TZVPP, accTZ=aug-cc-pVTZ, QZVPP=def2-QZVPP, SVP=def2-SVP).

\begin{tabular}{|l|c|c|c|c|c|c|c|c|c|c|}
\hline & \multicolumn{4}{|c|}{$G_{0} W_{O}$} & \multicolumn{5}{c|}{ evGW } \\
\cline { 2 - 12 } & $-\mathrm{IP}$ & $-\mathrm{EA}$ & $\Delta_{\mathrm{F}}$ & $\Delta_{\mathrm{O}}$ & $\mathrm{EBE}$ & $-\mathrm{IP}$ & $-\mathrm{EA}$ & $\Delta_{\mathrm{F}}$ & $\Delta_{\mathrm{O}}$ & EBE \\
\hline SR/TZVPP & -7.807 & -0.518 & 7.29 & 2.731 & 4.56 & -8.534 & -0.287 & 8.25 & 3.675 & 4.57 \\
\hline AC/TZVPP & -7.823 & -0.513 & 7.31 & 2.770 & 4.54 & -8.556 & -0.291 & 8.27 & 3.706 & 4.56 \\
\hline SR/accTZ* & -7.868 & -0.645 & 7.22 & 2.658 & 4.56 & -8.594 & -0.439 & 8.16 & 3.565 & 4.59 \\
\hline SR/QZVPP* & -8.028 & -0.645 & 7.38 & 2.831 & 4.55 & -8.790 & -0.430 & 8.36 & 3.784 & 4.58 \\
\hline SR/SVP & -7.452 & -0.385 & 7.07 & 2.491 & 4.58 & -8.137 & -0.145 & 7.99 & 3.405 & 4.59 \\
\hline AC/SVP & -7.465 & -0.378 & 7.09 & 2.530 & 4.56 & -8.142 & -0.146 & 8.00 & 3.422 & 4.57 \\
\hline
\end{tabular}

* Using the def2-TZVPP optimised geometry 
Table 2 Highest occupied (-IP) and lowest unoccupied (-EA) quasiparticle states, fundamental gap $\left(\Delta_{\mathrm{F}}\right)$, optical gap $\left(\Delta_{\mathrm{O}}\right)$ and exciton binding energy (EBE) values of $(\mathrm{MgO})_{32}$ as calculated by the different method combinations (TZVPP $=$ def2-TZVPP, $\mathrm{SVP}=$ def2-SVP). See table $\mathrm{S} 1$ in the supplementary information for $\mathrm{BSE} / G_{0} W_{0}(\mathrm{AC}) /$ def2-TZVPP data starting from PBE rather than B3LYP orbitals.

\begin{tabular}{|l|c|c|c|c|c|c|c|c|c|c|}
\hline & \multicolumn{9}{|c|}{$G_{0} W_{0}$} & \multicolumn{5}{c|}{ evGW } \\
\cline { 2 - 11 } & - IP & - EA & $\Delta_{\mathrm{F}}$ & $\Delta_{\mathrm{O}}$ & EBE & - IP & - EA & $\Delta_{\mathrm{F}}$ & $\Delta_{\mathrm{O}}$ & EBE \\
\hline SR/TZVPP & -7.320 & -0.365 & 6.96 & 3.710 & 3.24 & -7.975 & -0.145 & 7.83 & 4.538 & 3.29 \\
\hline AC/TZVPP & -7.333 & -0.361 & 6.97 & 3.810 & 3.14 & -8.009 & -0.152 & 7.86 & 4.680 & 3.18 \\
\hline SR/SVP & -6.989 & -0.172 & 6.82 & 3.531 & 3.29 & -7.602 & 0.057 & 7.66 & 4.320 & 3.34 \\
\hline AC/SVP & -7.001 & -0.167 & 6.83 & 3.691 & 3.14 & -7.618 & 0.051 & 7.67 & 4.494 & 3.18 \\
\hline
\end{tabular}

Second the effect of the different $G W$ approximations and implementations is considered. Going from $G_{0} W_{o}$ to evGW the highest occupied quasiparticle state moves to considerably deeper, more negative, values, the lowest unoccupied quasiparticle state to noticeably more shallow, less negative, values, the fundamental and optical gap increase significantly, while the exciton binding energy stays essentially the same. In contrast, as expected based on the literature, the variation of results calculated between the different $G W$ implementations is small. Similarly, the effect of going from evGW to full quasiparticle self-consistent qs $G W$ is small other than for the optical gap, which increases by $\sim 0.3 \mathrm{eV}$, and the exciton binding energy values, which decrease by a similar amount.

The optical gap of the $(\mathrm{MgO})_{4}$ particles was also calculated using coupled-cluster theory by LR-CCSD calculations. LR-CCSD predicts optical gap values of $3.90 \mathrm{eV}$ and $3.98 \mathrm{eV}$ when using the def2-TZVPP and def2-QZVPP basis-sets, respectively. Comparing these values to the results in Table 1 , shows that BSE $G_{0} W_{0}$ calculations irrespective of the basis-set used significantly underestimate the optical gap relative to LR-CCSD. In contrast, the BSE/evGW optical gap values lie much closer to their LRCCSD counterparts while BSE/qsGW essentially predicts the same values as LR-CCSD. It should be noted here that both the GW and LR-CCSD calculations ignore vibronic and zero-point motion effects and that as such this is a fair comparison but that the true experimental gap likely will be smaller as a result of such effects.

Based on the comparison with LR-CCSD, BSE/qsGW/def2-QZVPP would be the preferred method to study the electronic and optical properties of the $\mathrm{MgO}$ 
nanoparticles. However, that is in practice not tractable if one wants to study a large number of relatively large particles as the cost of the calculations scales strongly with the basis-set size and qs $G W$ is only implemented in Turbomole in combination with the most computationally expensive spectral representation of $G W$. Therefore, in the remainder mostly $G_{0} W_{0}$ and evGW calculations will be presented, including calculations with the def2-SVP basis-set. The effect of basis-set while significant is smaller than that of the $G W$ approximation used, optical gap values predicted by evGW/def2-SVP lie much closer to that predicted by LR-CCSD than $G_{0} W_{0} /$ def2-QZVPP. More importantly perhaps, all method combinations show the same changes when going from $(\mathrm{MgO})_{4}$ to $(\mathrm{MgO})_{32}$ and for each of the particles all method combinations predict very similar exciton binding energy values and hence a consistent balance between the fundamental and optical gaps.

Change in quasiparticle state energies and optical gap with particle size.

Table 3 gives the highest occupied and lowest unoccupied quasiparticle energies, fundamental gap, optical gap and exciton binding energy values predicted by $\mathrm{BSE} / G_{0} W_{0}(\mathrm{AC}) / \mathrm{def} 2-\mathrm{SVP}$ and BSE/evGW(AC)/def2-SVP for a series of cubic and cuboid $\mathrm{MgO}$ nanoparticles. Data is shown for $(\mathrm{MgO})_{4}$, the $n \times 4 \times 4$ family, including $(\mathrm{MgO})_{32}$, and the $n \times 5 \times 5$ family, which includes $(\mathrm{MgO})_{108}$.

Firstly, concentrating on the optical gap values, it can be observed that all particles studied, bar $(\mathrm{MgO})_{4}$, have essentially the same optical gap value of $3.6 \mathrm{eV}$ $\left(G_{0} W_{0}\right)$ or $4.1 \mathrm{eV}(\mathrm{evGW})$. Analysis of the responsible excitation in terms of the most significant natural transition orbitals shows that for all these particles this involves an excitation where an electron gets excited from the 3-coordinated oxygen corner atoms to the immediately adjacent 4-coordinated magnesium edge atoms (see Fig. 3A). The localised nature of the excitation and the fact that the surface motif responsible is present in all cuboid particles where all edges are at least two unit-cell lengths, probably explains the absence of variation in optical gap with particle size.

Table 3 Highest occupied (-IP) and lowest unoccupied (-EA) quasiparticle states, fundamental gap $\left(\Delta_{\mathrm{F}}\right)$, optical gap $\left(\Delta_{\mathrm{O}}\right)$ and exciton binding energy (EBE) values of the different cuboidal nanoparticles as predicted using BSE $/ G_{0} W_{0}(\mathrm{AC}) / \mathrm{def} 2-\mathrm{SVP}$ and BSE/evGW(AC)/def2-SVP. 


\begin{tabular}{|lc|c|c|c|c|c|c|c|c|c|c|}
\hline \multirow{2}{*}{$\mathrm{MgO}$} & \multicolumn{9}{|c|}{$G_{0} W_{\mathrm{O}}$} & \multicolumn{5}{|c|}{ evGW } \\
\cline { 3 - 13 } & & $-\mathrm{IP}$ & $-\mathrm{EA}$ & $\Delta_{\mathrm{F}}$ & $\Delta_{\mathrm{O}}$ & $\mathrm{EBE}$ & $-\mathrm{IP}$ & $-\mathrm{EA}$ & $\Delta_{\mathrm{F}}$ & $\Delta_{\mathrm{O}}$ & $\mathrm{EBE}$ \\
\hline 4 & $(2: 2: 2)$ & -7.311 & -0.416 & 6.90 & 2.362 & 4.53 & -7.984 & -0.181 & 7.80 & 3.253 & 4.55 \\
\hline 24 & $(3: 4: 4)$ & -6.802 & -0.363 & 6.44 & 3.574 & 2.86 & -7.429 & -0.139 & 7.29 & 4.395 & 2.90 \\
\hline 32 & $(4: 4: 4)$ & -6.904 & -0.209 & 6.70 & 3.599 & 3.10 & -7.524 & 0.015 & 7.54 & 4.409 & 3.13 \\
\hline 40 & $(5: 4: 4)$ & -6.680 & -0.229 & 6.63 & 3.601 & 3.03 & -7.476 & -0.008 & 7.47 & 4.403 & 3.06 \\
\hline 48 & $(6: 4: 4)$ & -6.863 & -0.200 & 6.66 & 3.604 & 3.06 & -7.479 & 0.023 & 7.50 & 4.407 & 3.09 \\
\hline 54 & $(4: 6: 6)$ & -6.535 & -0.532 & 6.00 & 3.626 & 2.38 & -7.160 & -0.306 & 6.85 & 4.441 & 2.41 \\
\hline 56 & $(7: 4: 4)$ & -6.853 & -0.198 & 6.66 & 3.606 & 3.05 & -7.468 & 0.025 & 7.49 & 4.407 & 3.09 \\
\hline 64 & $(8: 4: 4)$ & -6.854 & -0.189 & 6.67 & 3.609 & 3.06 & -7.469 & 0.035 & 7.50 & 4.412 & 3.09 \\
\hline 72 & $(4: 6: 6)$ & -6.823 & -0.206 & 6.62 & 3.618 & 3.00 & -7.434 & 0.017 & 7.45 & 4.415 & 3.04 \\
\hline 90 & $(5: 6: 6)$ & -6.650 & -0.340 & 6.31 & 3.607 & 2.70 & -7.262 & -0.119 & 7.14 & 4.402 & 2.70 \\
\hline 108 & $(6: 6: 6)$ & -6.703 & -0.246 & 6.46 & 3.597 & 2.86 & -7.313 & -0.022 & 7.29 & 4.392 & 2.90 \\
\hline
\end{tabular}

In contrast to the optical gap, the quasiparticle state energies and by extension also the fundamental gap do change with particle size, even if the picture is confusing. Focussing first on the cubes: $(\mathrm{MgO})_{4},(\mathrm{MgO})_{32}$ and $(\mathrm{MgO})_{108}$, it can be observed that the highest occupied quasiparticle state consistently moves to less negative, shallower, values. The lowest unoccupied quasiparticle state also moves to less negative, shallower, values when going from $(\mathrm{MgO})_{4}$ to $(\mathrm{MgO})_{32}$ but then moves to slightly more negative, deeper, values when going to $(\mathrm{MgO})_{108}$. The fundamental gap, finally, consistently gets smaller with increasing edge length and particle size.

For the cuboid $n \times 4 \times 4$ and $n \times 5 \times 5$ families, where $n$ is even, increasing the length of one of the edges, e.g. going from $(\mathrm{MgO})_{32}$ to $(\mathrm{MgO})_{48}$, shift the highest occupied quasiparticle state to less negative values, and decreasing the length of one of the edges, e.g. going from $(\mathrm{MgO})_{108}$ to $(\mathrm{MgO})_{72}$, shift the highest occupied quasiparticle state to more negative values. The lowest unoccupied quasiparticle state shifts to less negative values with increasing edge length for the $n \times 3 \times 3$ family but, just like for the cubes, shifts to more negative values for the $n \times 5 \times 5$ family. For the $n$ is odd cuboids, which differ from the cubes and the $n$ is even cuboids in the fact that the corner atoms of the edge are the same (e.g. both oxygen atoms) rather than different, similar trends can be observed but the absolute values appear shifted. Just as for the cubes the fundamental gap for the different cuboid families appears to decrease with particle size and edge length. For the highest occupied and lowest unoccupied quasiparticle states and the fundamental gap, finally, the effect of increasing the edge length appears to decrease in magnitude when one edge becomes much longer than the other two. 
A

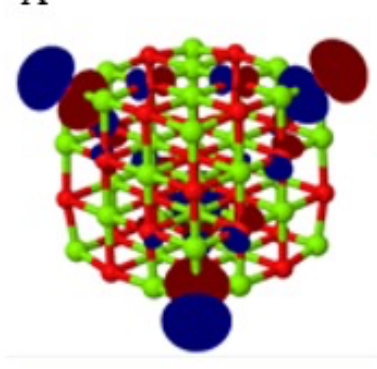

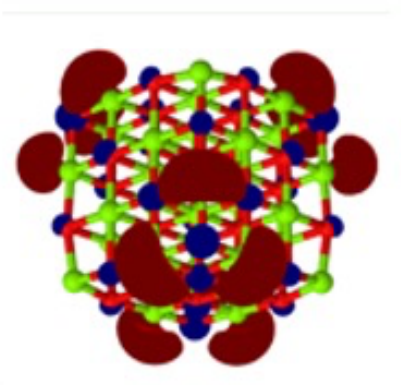

B
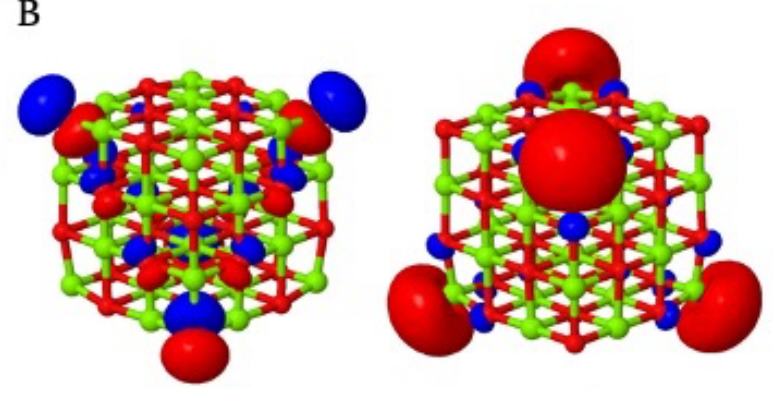

Fig. 3 Leading occupied (A, left) and unoccupied (A, right) natural transition orbitals for the lowest excitation of $(\mathrm{MgO})_{32}$ and the highest occupied (B, left) and lowest unoccupied (B, right) Kohn-Sham orbitals for the same particle. As the calculation was performed using the $D_{2}$ instead of the $T_{\mathrm{d}}$ point group, the triply degenerate $\mathrm{T}_{2}$ excited state is described as a triplet of excited states of $\mathrm{B}_{1}, \mathrm{~B}_{2}$ and $\mathrm{B}_{3}$ symmetry, the natural transition orbitals of only one is shown in A. Similarly (B, left) is one of three degenerate orbitals.

While the lowest excitation corresponding to the optical gap for all particles involves, as discussed above, the 3-coordinated oxygen corner atoms and the immediately adjacent 4-coordinated magnesium edge atoms, the ground-state KohnSham orbitals from DFT corresponding to the highest occupied and lowest unoccupied quasiparticle states for all cubic and cuboid particles considered are localised on the 3coordinated oxygen atoms, with minor contributions of the oxygen atoms on the edge or diagonal between these 3-coordinated oxygen atoms, and 3-coordinated magnesium atoms, respectively (see Fig. 3B). The fact that in the lowest excitation the electron gets excited from 4-coordinated magnesium edge atoms adjacent to the 3-coordinated oxygen corner atoms rather than from the 3-coordinated magnesium corner atoms combined with the fact that the optical gap is much smaller than the fundamental gap for all particles suggests that this excitation is excitonic in character. 
Table 4 Highest occupied (-IP) and lowest unoccupied (-EA) quasiparticle states, fundamental gap $\left(\Delta_{\mathrm{F}}\right)$, optical gap $\left(\Delta_{\mathrm{O}}\right)$ and exciton binding energy (EBE) values of the different cuboidal nanoparticles with odd-membered faces as predicted using $\mathrm{BSE} / G_{0} W_{0}(\mathrm{AC}) / \mathrm{def} 2-\mathrm{SVP}$ and BSE/evGW(AC)/def2-SVP.

\begin{tabular}{|c|c|c|c|c|c|c|c|c|c|c|}
\hline \multirow[t]{2}{*}{$\mathrm{MgO}$} & \multicolumn{5}{|c|}{$G_{0} W_{0}$} & \multicolumn{5}{|c|}{ evGW } \\
\hline & -IP & -EA & $\Delta_{\mathrm{F}}$ & $\Delta_{\mathrm{O}}$ & EBE & -IP & -EA & $\Delta_{\mathrm{F}}$ & $\Delta_{\mathrm{O}}$ & EBE \\
\hline$(3: 3: 3)$ & -6.362 & $\begin{array}{c}-0.772 \\
\end{array}$ & 5.59 & 3.278 & 2.31 & -6.998 & -0.548 & 6.45 & 4.127 & 2.32 \\
\hline$(4: 3: 3)$ & $\begin{array}{l}-5.817 \\
\end{array}$ & -1.225 & 4.59 & 3.082 & 1.51 & -6.451 & -0.997 & 5.45 & 3.950 & 1.50 \\
\hline
\end{tabular}

Cuboid MgO particles with odd-numbered faces.

Table 4 gives the highest occupied and lowest unoccupied quasiparticle energies, fundamental gap, optical gap and exciton binding energy values predicted by $\mathrm{BSE} / G_{0} W_{0}(\mathrm{AC}) / \operatorname{def} 2-\mathrm{SVP}$ and BSE/evGW(AC)/def2-SVP for a series of cuboid $\mathrm{MgO}$ nanoparticles of which two of the faces have an odd number of atoms. The same results calculated with def2-TZVPP for the smallest one $\left((\mathrm{MgO})_{18}\right)$ can be found in Table S2 in the supplementary material. In the previous (TD-)DFT study of $\mathrm{MgO}$ nanoparticles it was noted that these odd-numbered faces behaved as if polar, even if infinitely extended (100) surfaces are not polar in the conventional sense. ${ }^{31}$ This polarity is the direct result of the particles exhibiting odd-membered faces. These particles can be thought of as stacking sequences of charged layers with odd number of atoms, where the odd-menbered faces of the particle are the outer most layers of the stack. The layers contain alternatingly either one more oxygen atom or one more magnesium atom, alternating $\left[\mathrm{Mg}_{5} \mathrm{O}_{4}\right]^{2+}$ and $\left[\mathrm{Mg}_{4} \mathrm{O}_{5}\right]^{2-}$ layers in the case of the specific particles studied here. This alternation of charged layers gives rise to a very large dipole moment along the stacking axis, normal to the odd-membered faces, (17.3 Debye and 32.6 Debye for $(\mathrm{MgO})_{18}$ and $(\mathrm{MgO})_{27}$, respectively), with the magnitude of the dipole increasing with the particle dimension along that axis. There is analogy to so-called Tasker type 3 surfaces $^{58}$ other than in that case the infinitely extended surfaces are inherently polar and here they are not. This polarisation in the ground state goes together with a reduction in the optical and fundamental gap of these particles relative to cubes and cuboidal particles lacking these odd-membered faces, where the size of both gaps decreases with the particle dimension along the axis of the particle perpendicular to 
the odd-membered faces. Analysis of the ground state orbitals from DFT and the most significant natural transition orbitals from BSE for $(\mathrm{MgO})_{27}$ clearly show the polarisation. The ground state orbital corresponding to the highest occupied quasiparticle state is localised on the central oxygen atom and the four oxygen corner atoms of the oxygen-rich odd-membered face and the orbital for the lowest unoccupied quasiparticle state is localised over the four magnesium corner atoms and the central magnesium atom of the magnesium-rich odd-membered face, while the most significant natural transition orbitals for the optical gap corresponds to an excitation of an electron from the oxygen atoms of the oxygen-rich odd membered face to the magnesium atoms of the magnesium rich face.

While particles with odd-membered faces larger than $(\mathrm{MgO})_{27}$ were not considered here, the data suggests that for very large particle dimension the fundamental and optical gaps of such particles might go to zero with the particles becoming metallic. However, as discussed below this situation is unlikely to be ever encountered in experiment.

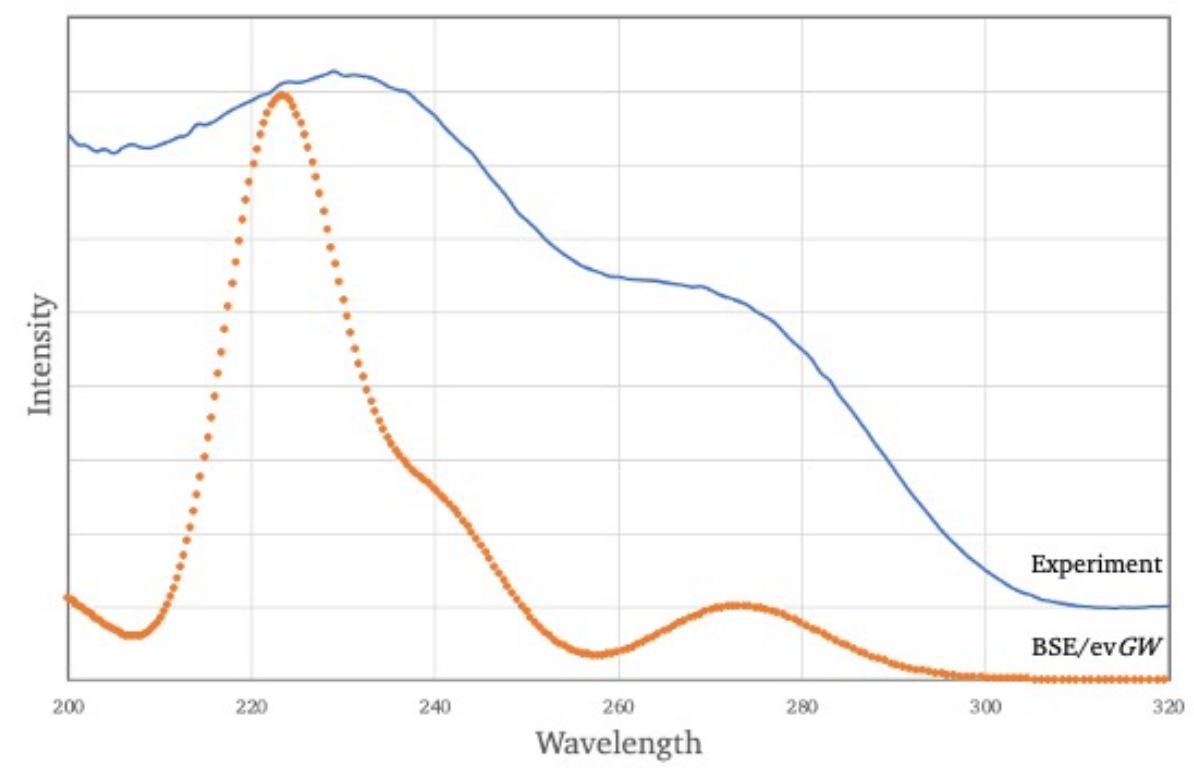

Fig. 4 The vertical excitation spectrum of $(\mathrm{MgO})_{32}$ as predicted using BSE/evGW(SR)/def2-TZVPP (red line) and the experimental measured reflection spectrum of $3 \mathrm{~nm} \mathrm{MgO}$ particles (blue line). Experimental data taken from reference 10. 
Optical spectra of cubic $\mathrm{MgO}$ particles.

Fig. 4 shows the vertical excitation spectrum of $(\mathrm{MgO})_{32}$ as predicted using

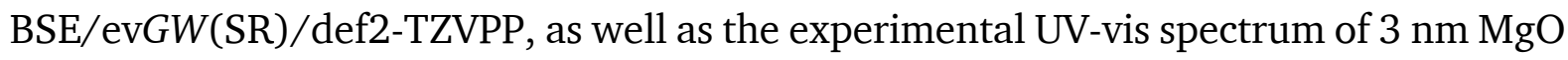
particles ${ }^{10}$ measured by Stankic and co-workers. While the roughly four times difference in size there is a good match between the predicted and experimental spectrum, in line with the observation above that the energy of the lowest exciton is the same for all cuboid particles with even faces and all edges corresponding to at least two unit-cell lengths. All excitations shown in Fig. 4 are 4.6-1.9 eV smaller than the evGW(SR)/def2TZVPP predicted fundamental gap, suggesting that not only the lowest energy / longest wavelength excitation but all excitations to at least $200 \mathrm{~nm}$ are excitonic in character.

The bright excitations were analysed in terms of the most important natural transition orbitals. The lowest bright excitation at $4.54 \mathrm{eV}$ or $273 \mathrm{~nm}$, responsible for the most red-shifted broad peak is, as discussed above, due to an excitation of an electron from the 3-coordinated oxygen corner atoms to the immediately adjacent 4coordinated magnesium edge atoms (See Fig. 3A). The shoulder at $240 \mathrm{~nm}$ is the sum of two bright excitations. The bright excitation at $5.12 \mathrm{eV}$ or $242 \mathrm{~nm}$ corresponds to an excitation of an electron from the 4-coordinated oxygen edge atoms to 3-coordinated corner magnesium atoms (see Fig. 5A), while the bright excitation at 5.17 or $240 \mathrm{~nm}$ corresponds just like the lowest bright excitation to an excitation of an electron from the 3-coordinated oxygen corner atoms to the adjacent 4-coordinated magnesium edge atoms (see Fig. 5B). The two main contributions to the strongest peak at $220 \mathrm{~nm}$ are an excitation at $5.52 \mathrm{eV}$ or $224 \mathrm{~nm}$, corresponding just like the $5.12 \mathrm{eV}$ or $242 \mathrm{~nm}$ excitation to an excitation of an electron from the 4-coordinated oxygen edge atoms to 3-coordinated corner magnesium atoms (see Fig. 5C), and an excitation at $5.59 \mathrm{eV}$ or $222 \mathrm{~nm}$, which involves a similar excitation of an electron from now both the 4coordinated edge and 6-coordinated bulk oxygen atoms to the 3-coordinated corner magnesium atoms (see Fig. 5D). Higher-energy shorter-wavelength bright excitations up to $200 \mathrm{~nm}$ display increased contribution of 5- and 6-coordinated oxygen atoms and 4-coordinated magnesium atoms but the 3-/4-coordinated oxygen atoms and 3coordinated magnesium atoms remain (the) most significant contributors. 
Compared to the previous TD-DFT calculations ${ }^{31}$ using the range-separated CAM-B3LYP functional the main difference is the predicted character of the excitations responsible for the $240 \mathrm{~nm}$ shoulder. TD-CAM-BLYP predicts that one of the excitations contributing to that shoulder corresponds to an excitation of an electron from a 3coordinated corner oxygen atom to a 3-coordinated corner magnesium atom, something that is not observed in the BSE/evGW predicted spectra to much higher excitation energies / shorter wavelengths.

A

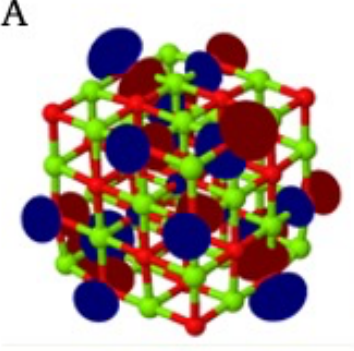

C

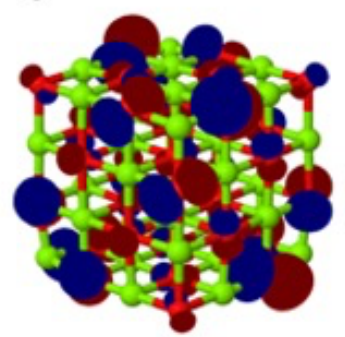

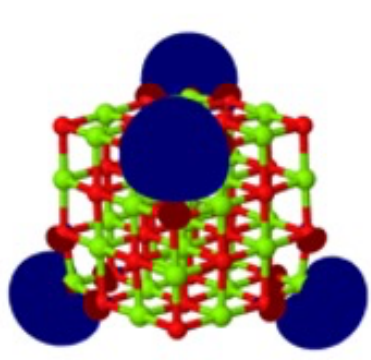

B
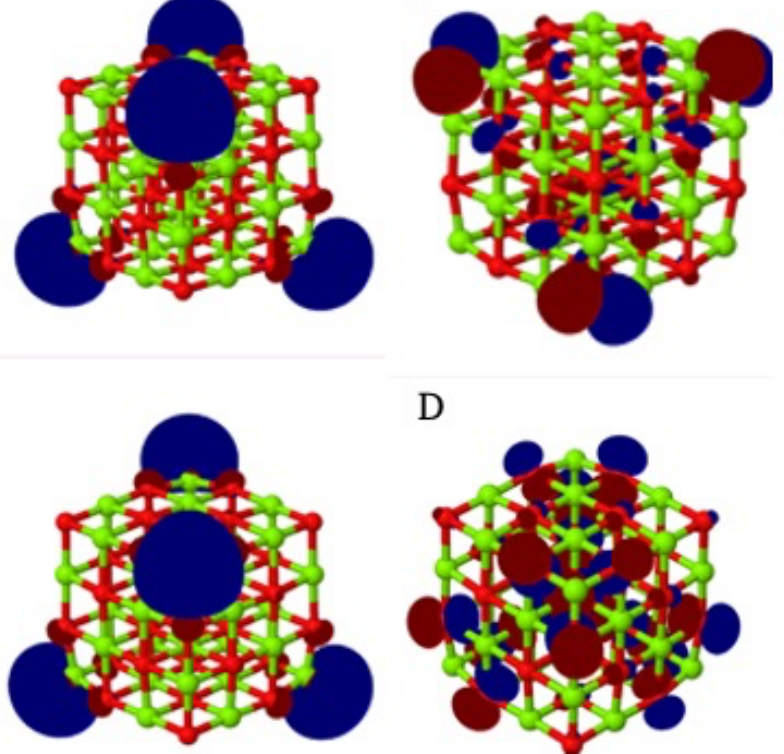

$\mathrm{D}$

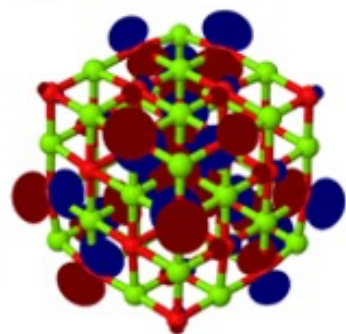

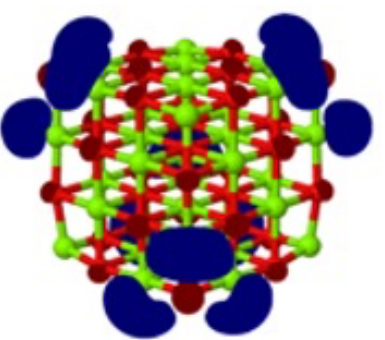

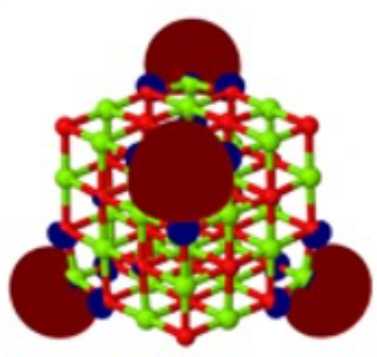

Fig. 5 Leading natural transition orbitals for the 5.12 (A), 5.17 (B), 5.52 (C) and 5.59 (D) eV bright excitations of $(\mathrm{MgO})_{32}$. Similar to Fig. 3. only one of the three degenerate leading natural transition orbitals for each of the excitations is shown.

Beyond $\mathrm{MgO}$

As discussed in the introduction nanoparticles of $\mathrm{CaO}$ and $\mathrm{SrO}$ grown through chemical vapour deposition show experimentally the same red-shift relative to the bulk in the $\mathrm{UV}$-Vis reflection spectra as $\mathrm{MgO}$ nanoparticles. To probe if nanoparticles of such other rocksalt materials display the same physics as that discussed above for $\mathrm{MgO}$ nanoparticles the optical and electronic properties of $4 \times 4 \times 4(\mathrm{CaO})_{32}$ and $(\mathrm{SrO})_{32}$ cubes were calculated using BSE/ $G_{0} W_{0}$ and $\mathrm{BSE} / \mathrm{ev} G W$, see Table 5. 
As can be seen from Table 5, the lowest excitations of these $\mathrm{CaO}$ and $\mathrm{SrO}$ particles are just as for their $\mathrm{MgO}$ counterparts excitonic in character with predicted exciton binding energies of more than $2 \mathrm{eV}$. Visualisation of the natural transition orbitals for these particles also show that, just in the case of $\mathrm{MgO}$ particles, the lowest energy excitons are strongly localised around the corner atoms, see Fig. S1 in the supporting information. Finally, the predicted optical gap values are comparable to experimentally measured lowest excitation energies of $\mathrm{CaO}(3.5 \mathrm{eV})^{11}$ and $\mathrm{SrO}(3.7 \mathrm{eV})^{12}$ nanoparticles.

Table 5 Highest occupied (-IP) and lowest unoccupied (-EA) quasiparticle states, fundamental gap $\left(\Delta_{\mathrm{F}}\right)$, optical gap $\left(\Delta_{\mathrm{O}}\right)$ and exciton binding energy (EBE) values of $(\mathrm{CaO})_{32}$ and $(\mathrm{SrO})_{32}$ cubes predicted using $\mathrm{BSE} / G_{0} W_{0}(\mathrm{AC}) /$ def2-TZVPP and BSE/evGW(AC)/def2-TZVPP.

\begin{tabular}{|l|c|c|c|c|c|c|c|c|c|c|}
\hline & \multicolumn{4}{|c|}{$G_{0} W_{0}$} & \multicolumn{5}{c|}{ evGW } \\
\cline { 2 - 12 } & $-\mathrm{IP}$ & $-\mathrm{EA}$ & $\Delta_{\mathrm{F}}$ & $\Delta_{\mathrm{O}}$ & $\mathrm{EBE}$ & $-\mathrm{IP}$ & $-\mathrm{EA}$ & $\Delta_{\mathrm{F}}$ & $\Delta_{\mathrm{O}}$ & EBE \\
\hline $\mathrm{CaO}(4: 4: 4)$ & -5.810 & -0.015 & 5.80 & 3.079 & 2.72 & -6.363 & 0.120 & 6.48 & $3.736^{*}$ & 2.75 \\
\hline $\mathrm{SrO}(4: 4: 4)$ & -5.270 & -0.042 & 5.23 & 2.640 & 2.59 & -5.805 & 0.086 & 5.89 & 3.278 & 2.61 \\
\hline
\end{tabular}

The lowest excited-state for $(\mathrm{CaO})_{32}$ as calculated using BSE/evGW(AC)/def2-TZVPP is symmetry forbidden. The $3.736 \mathrm{eV}$ excitation is the lowest optically allowed excitation, second lowest overall. The lowest BSE/evGW(AC)/def2-TZVPP excitation belongs to the A irrep for $T_{\mathrm{d}}$ and has an excitation energy of $3.634 \mathrm{eV}$ and an EBE value of $2.85 \mathrm{eV}$.

\section{Discussion}

The BSE/evGW calculations show in line with the experimental work by Stankic and others that the optical gap of $\mathrm{MgO}$ nanoparticles is much smaller than that of the bulk and that such particles absorb light in the middle ultraviolet, 200-300 nm, where the bulk does not. The excitations responsible for the light absorption in that wavelength range are all predicted to be excitonic in character as the predicted fundamental gap of all particles considered, excluding those with odd-membered faces, is larger than 200 $\mathrm{nm}$. The lowest exited-state in all cases bar the smallest nanoparticles corresponds to a well-localised exciton involving the 3-coordinated oxygen corner atoms and their immediate 4-coordinated magnesium atoms, where the optical gap does not change with particle size. These $\mathrm{MgO}$ nanoparticles hence, as suggested by previous embedded cluster calculations discussed above, behave as defective insulators, where light absorption at longer wavelengths / lower energies than the bulk is due to excitations of electrons between what are essentially surface defects, rather than quantum dots. 
As discussed above the quasiparticle states and the fundamental gap in contrast to the optical gap do change with particle size. The relevant orbitals are again relatively localised but more delocalised than the excited state associated with the optical gap. The origin of the variation of these electronic properties with particle size is unknown. Perhaps more interestingly is the fact that the nature of the variation is unexpected. The highest occupied quasiparticle state is found to move to shallower values, the lowest unoccupied quasiparticle state to deeper values and hence the fundamental gap decreases with particle size. The bulk fundamental gap cannot be calculated with the same exact set-up as used here for the particles. However, literature prediction of the fundamental gap of bulk $\mathrm{MgO}$ using $G_{0} W_{0}$ range from 7.5-8 $\mathrm{eV}^{59-63}$ and for qs $G W$ a value of $8.69 \mathrm{eV}$ has been reported by Lambrecht and co-workers ${ }^{64}$ (in all cases, like the $G W$ calculations here, ignoring vibronic and zero point motion effects, the inclusion of the former brings the $\mathrm{qs} G W$ values for the bulk closer to the experimental value of $7.8 \mathrm{eV}^{65-}$ $\left.{ }^{66}\right)$. Comparing these bulk values from the literature to the $G_{0} W_{o}$ and evGW reported here for the nanoparticles here than the nanoparticles (i) have smaller fundamental gaps than bulk $\mathrm{MgO}$, (ii) the nanoparticle highest occupied and lowest unoccupied quasiparticle states likely lie within the fundamental gap of bulk $\mathrm{MgO}$, and (iii) that upon increasing the particle size the fundamental gap appears to diverge away from the bulk value rather than converge towards it. While these observations are by definition is based on a limited range of particle sizes, e.g. the next cube has $232 \mathrm{MgO}$ formula units and hence for the moment beyond the horizon of what can be achieved with the $G W$ implementation, it suggests that even for (macroscopically) large particles the corner atoms fix the highest occupied and lowest unoccupied quasiparticle states and the fundamental gap. That is even if this is experimentally unobservable by photoelectron spectroscopy due the relatively very small number of such corner atoms in large particles.

It turns out to be impossible to predict the $(\mathrm{MgO})_{108}$ spectrum using $\mathrm{BSE} / \mathrm{evGW}$, even when using the def2-SVP basis-set, due to the very large memory and disk-space requirements to calculate more than just the lowest excited states for this particle. However, it stands to reason that the observed trend in the intensities of the peaks in the experimental reflection spectra, where going from 3 to $10 \mathrm{~nm}$ particles the relative 
intensity of the 270 and $240 \mathrm{~nm}$ shoulders/peaks decreases and that of $220 \mathrm{~nm}$ peak increases, finds its origin in the fact that the most red-shifted peaks involve excitations localised on exclusively 3-coordinated corner and 4-coordinated edge atoms while the $220 \mathrm{~nm}$ peak also involve 5-coordinated terrace and 6-coordinated bulk atoms and that when increasing the particle size the number of corner and edge atoms relative to surface and bulk atoms decreases. For the same reason and in analogy to the discussion above for the fundamental gap, even for macroscopically large $\mathrm{MgO}$ particles the true optical gap would likely be the same as that predicted here for nanoparticles but the optical gap observed in experiment would probably be the bulk value.

The exciton binding energy decreases with particle size, driven by the reduction in the fundamental gap with particle size. In the case of pure cubes extrapolation to the infinite particle limit by plotting the BSE/evGW/def2-SVP exciton binding energy of the lowest energy exciton of the three cubes against the inverse of their edge lengths yields a value of $2.4 \mathrm{eV}$. This value is more than an order of magnitude larger than the binding energy of the $\mathrm{n}=1$ bulk exciton, $80 \mathrm{meV},{ }^{65-66}$ which is in line with the former probably ${ }^{67}$ being a Wannier-Mott exciton while the lowest exciton in the nanoparticles, as discussed, is much more localised and hence more like a Frenkel exciton.

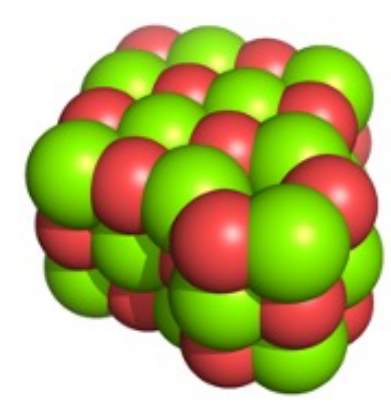

Fig. 6 Structure of the alternative structure considered for $(\mathrm{MgO})_{27}$, the global minimum as proposed by Dixon and co-workers. ${ }^{21}$

The nanoparticles with odd-membered faces have rather different optical and electronic properties relative to their counterparts that only have even-membered faces. However, particles displaying such odd-membered surfaces are unlikely to be observed in experiment as the same ground-state dipole moment that gives rise to these different optical and electronic properties also destabilises them energetically. Previous global 
optimisation studies find that while for $(\mathrm{MgO})_{18}$ the cuboidal rocksalt structure is still the predicted global minimum, as it is for particles with only even-membered faces (i.e. $(\mathrm{MgO})_{24},(\mathrm{MgO})_{32}$ and $\left.(\mathrm{MgO})_{40}\right)$, for $(\mathrm{MgO})_{27}$ a reconstructed cuboidal structure with a much reduce ground-state dipole moment ( 0.3 instead of 32.6 Debye) is found to be more stable ${ }^{21}$ (see Fig. 6). As expected this reconstructed ( $\left.\mathrm{MgO}\right)_{27}$ particle also has a larger optical gap $(4.48 \mathrm{eV}$ instead of $3.95 \mathrm{eV}$ when calculated with $\mathrm{BSE} / \mathrm{evGW}(\mathrm{AC}) / \mathrm{def} 2-\mathrm{SVP}$, see table S3 in the supporting information), which is similar to that of the cubic particles with even-membered faces. In analogy to classically polar surfaces, these particles with odd-membered surfaces can thus be thought to reconstruct to eliminate the large dipole moment and in the process also eliminate their more out of kilter optical properties.

Finally, while this paper studies primarily $\mathrm{MgO}$ nanoparticles, the same physics is likely in play in nanoparticles of other oxides. Indeed, as mentioned in the introduction nanoparticles of $\mathrm{CaO}$ and $\mathrm{SrO}$ have experimentally been observed to display the same red-shift relative to the bulk as $\mathrm{MgO}$ nanoparticles and $\mathrm{BSE} / \mathrm{GW}$ calculations discussed above confirm that for these materials the lowest energy excitations are excitonic in nature and strongly localised around the corner atoms of the nanoparticles. Comparing the BSE/evGW predicted optical gap for the nanoparticles with the experimental excitation energy of the lowest exciton peak in bulk $\mathrm{CaO}(7 \mathrm{eV})^{68}$ and $\mathrm{SrO}(5.7 \mathrm{eV}){ }^{69-71}$ confirms the bulk to nanoparticle red shift for these materials. As noted above for $\mathrm{MgO}$, an exact one-to-one comparison to properties predicted for the bulk is difficult, but the $\mathrm{BSE} / G_{0} W_{0} / \mathrm{PBE}$ optical gap values predicted for the $\mathrm{CaO}$ and $\mathrm{SrO}$ nanoparticles (see table $\mathrm{S} 4$ ) are also indeed significantly smaller than those extracted from the results of periodic BSE $/ G_{0} W_{0} / \mathrm{PBE}$ calculations from the literature. ${ }^{62,72}$ Interestingly, a comparison of the fundamental gap predicted for bulk $\mathrm{CaO}^{62,73}$ and $\mathrm{SrO}^{72-73}$ using $G_{0} W_{0} / \mathrm{PBE}$, and the equivalent fundamental gap of the nanoparticles shows that they are more similar than for $\mathrm{MgO}$, especially in the case of SrO, for which the $G_{0} W_{0} / \mathrm{PBE}$ fundamental gap ( $4.85 \mathrm{eV}$ ) is virtually identical to that predicted for the bulk. While the lowest energy excitons for nanoparticles of these materials remain clearly localised around the particles' corner atoms and the optical 
properties thus governed by the presence of these low-coordinated atoms, the highest occupied and lowest unoccupied quasiparticle states appear less tied down.

\section{Conclusions}

evGW-BSE calculations on realistic oxide nanoparticles such as those made from $\mathrm{MgO}$ are found to be computationally tractable and using such calculations it is demonstrated that the optical and electronic properties of $\mathrm{MgO}$ nanoparticles are governed by the presence of low-coordinated atoms on their surfaces, in other words are surface defect states. States localised on these corner and edge atoms are responsible for the highest occupied and lowest unoccupied quasiparticle states, i.e. the ionisation potential and the electron affinity, of the particle, and thus the magnitude of the fundamental gap, as well as the lowest excited states and thus the size of the optical gap. As a result the optical gap of all cuboidal rocksalt $\mathrm{MgO}$ nanoparticles bar the absolute smallest and particles with odd membered faces is predicted to be essentially the same and to not vary with the particle size. By extrapolation it can be assumed that the same holds true for macroscopic $\mathrm{MgO}$ particles, even if this might be hard to observe experimentally due to the much smaller surface to volume ratio for such particles. The $\mathrm{BSE} / \mathrm{evGW}$ spectrum predicted for $(\mathrm{MgO})_{32}$ agrees well with the experimental spectra for $\mathrm{MgO}$ nanoparticles reported in the literature.

Nanoparticles with odd membered faces are predicted to have reduced optical gaps and display large ground state dipole moments perpendicular to the odd membered faces. The presence of such large dipole moments destabilises these particles relative to particles that lack odd membered faces and hence can be argued to reconstruct to reduce the dipole moments, akin to what happens in the case of polar surfaces.

Finally, calculations on $\mathrm{CaO}$ and $\mathrm{SrO}$ nanoparticles show that they display the same physics as $\mathrm{MgO}$ nanoparticles with the lowest energy excitations corresponding to excitons localised on the particles' corners. Nanoparticles of $\mathrm{MgO}$ are thus not unique in having electronic and optical properties governed by surface states instead of quantum confinement effects. In fact, the behaviour observed for $\mathrm{MgO}$ appears typical of nanoparticles of all alkaline earth oxides and perhaps many other oxides. 


\section{Acknowledgements}

Drs. Uwe Hunlar, Christof Holzer, Johannes Lischner and Slavica Stankic are kindly acknowledged for discussion. The UK Engineering and Physical Sciences Research Council (EPSRC) is thanked for funding part of this work through grants EP/I004424/1 and EP/N004884/1.

\section{References}

1. Efros, A. L.; Brus, L. E., Nanocrystal Quantum Dots: From Discovery to Modern Development. ACS Nano 2021, 10.1021/acsnano.1c01399.

2. Brus, L., Electronic Wave Functions in Semiconductor Clusters: Experiment and Theory. The Journal of Physical Chemistry 1986, 90, 2555-2560.

3. Brus, L. E., Electron-Electron and Electron-Hole Interactions in Small Semiconductor Crystallites: The Size Dependence of the Lowest Excited Electronic State. The Journal of Chemical Physics 1984, 80, 4403-4409.

4. Yu, W. W.; Peng, X., Formation of High-Quality CdS and Other II-VI Semiconductor Nanocrystals in Noncoordinating Solvents: Tunable Reactivity of Monomers. Angewandte Chemie International Edition 2002, 41, 2368-2371.

5. Murray, C. B.; Norris, D. J.; Bawendi, M. G., Synthesis and Characterization of Nearly Monodisperse CdE (E = Sulfur, Selenium, Tellurium) Semiconductor Nanocrystallites. Journal of the American Chemical Society 1993, 115, 8706-8715.

6. Peng, X.; Wickham, J.; Alivisatos, A. P., Kinetics of II-VI and III-V Colloidal Semiconductor Nanocrystal Growth: "Focusing" of Size Distributions. Journal of the American Chemical Society 1998, 120, 5343-5344.

7. Cademartiri, L.; Montanari, E.; Calestani, G.; Migliori, A.; Guagliardi, A.; Ozin, G. A., Size-Dependent Extinction Coefficients of PbS Quantum Dots. Journal of the American Chemical Society 2006, 128, 10337-10346.

8. Hines, M. A.; Scholes, G. D., Colloidal PbS Nanocrystals with Size-Tunable near-Infrared Emission: Observation of Post-Synthesis Self-Narrowing of the Particle Size Distribution. Advanced Materials 2003, 15, 1844-1849.

9. Murray, C. B.; Sun, S.; Gaschler, W.; Doyle, H.; Betley, T. A.; Kagan, C. R., Colloidal Synthesis of Nanocrystals and Nanocrystal Superlattices. IBM J. Res. Dev. 2001, 45, 47-56.

10. Stankic, S.; Müller, M.; Diwald, O.; Sterrer, M.; Knözinger, E.; Bernardi, J., SizeDependent Optical Properties of $\mathrm{MgO}$ Nanocubes. Angewandte Chemie International Edition 2005, 44, 4917-4920.

11. Stankic, S.; Bernardi, J.; Diwald, O.; Knözinger, E., Optical Surface Properties and Morphology of MgO and CaO Nanocrystals. The Journal of Physical Chemistry B 2006, 110, 13866-13871.

12. Stankic, S.; Bernardi, J.; Diwald, O.; Knözinger, E., Photoexcitation of Local Surface Structures on Strontium Oxide Grains. The Journal of Physical Chemistry C 2007, 111, 80698074. 
13. Cohen, M. L.; Lin, P. J.; Roessler, D. M.; Walker, W. C., Ultraviolet Optical Properties and Electronic Band Structure of Magnesium Oxide. Physical Review 1967, 155, 992-996.

14. Roessler, D. M.; Walker, W. C., Spin-Orbit Splitting of the Gamma Exciton in MgO. Physical Review Letters 1966, 17, 319-320.

15. Spanó, E.; Hamad, S.; Catlow, C. R. A., Computational Evidence of Bubble ZnS Clusters. The Journal of Physical Chemistry B 2003, 107, 10337-10340.

16. Spanó, E.; Hamad, S.; Catlow, C. R. A., ZnS Bubble Clusters with Onion-Like Structures. Chemical Communications 2004, 864-865.

17. Hamad, S.; Catlow, C. R. A.; Spanó, E.; Matxain, J. M.; Ugalde, J. M., Structure and Properties of ZnS Nanoclusters. The Journal of Physical Chemistry B 2005, 109, 2703-2709.

18. Sanville, E.; Burnin, A.; BelBruno, J. J., Experimental and Computational Study of Small $(\mathrm{N}=1-16)$ Stoichiometric Zinc and Cadmium Chalcogenide Clusters. The Journal of Physical Chemistry A 2006, 110, 2378-2386.

19. Zwijnenburg, M. A., Optical Excitations in Stoichiometric Uncapped ZnS Nanostructures. Nanoscale 2011, 3, 3780-3787.

20. Roberts, C.; Johnston, R. L., Investigation of the Structures of MgO Clusters Using a Genetic Algorithm. Physical Chemistry Chemical Physics 2001, 3, 5024-5034.

21. Chen, M.; Felmy, A. R.; Dixon, D. A., Structures and Stabilities of $(\mathrm{MgO})_{\mathrm{N}}$ Nanoclusters. The Journal of Physical Chemistry A 2014, 118, 3136-3146.

22. Escher, S. G. E. T.; Lazauskas, T.; Zwijnenburg, M. A.; Woodley, S. M., Synthesis Target Structures for Alkaline Earth Oxide Clusters. Inorganics 2018, 6, 29.

23. Trevisanutto, P. E.; Sushko, P. V.; Shluger, A. L.; Beck, K. M.; Henyk, M.; Joly, A. G.; Hess, W. P., A Mechanism of Photo-Induced Desorption of Oxygen Atoms from MgO NanoCrystals. Surface Science 2005, 593, 210-220.

24. Müller, M.; Stankic, S.; Diwald, O.; Knözinger, E.; Sushko, P. V.; Trevisanutto, P. E.; Shluger, A. L., Effect of Protons on the Optical Properties of Oxide Nanostructures. Journal of the American Chemical Society 2007, 129, 12491-12496.

25. McKenna, K. P.; Koller, D.; Sternig, A.; Siedl, N.; Govind, N.; Sushko, P. V.; Diwald, O., Optical Properties of Nanocrystal Interfaces in Compressed MgO Nanopowders. ACS Nano 2011, 5, 3003-3009.

26. Sternig, A.; Koller, D.; Siedl, N.; Diwald, O.; McKenna, K., Exciton Formation at SolidSolid Interfaces: A Systematic Experimental and Ab Initio Study on Compressed MgO Nanopowders. The Journal of Physical Chemistry C 2012, 116, 10103-10112.

27. Wu, M.-C.; Truong, C. M.; Goodman, D. W., Electron-Energy-Loss-Spectroscopy Studies of Thermally Generated Defects in Pure and Lithium-Doped $\mathrm{MgO}(100)$ Films on Mo(100). Physical Review B 1992, 46, 12688-12694.

28. Kolmakov, A.; Stultz, J.; Goodman, D. W., Characterization of Surface Defects on MgO Thin Films by Ultraviolet Photoelectron and Metastable Impact Electron Spectroscopies. The Journal of Chemical Physics 2000, 113, 7564-7570.

29. Cox, P. A.; Williams, A. A., Surface Excitons on Ionic Crystals. Surface Science Letters 1986, 175, L782-L786.

30. Rohlfing, M.; Wang, N.-P.; Krüger, P.; Pollmann, J., Image States and Excitons at Insulator Surfaces with Negative Electron Affinity. Physical Review Letters 2003, 91, 256802.

31. Wobbe, M. C. C.; Kerridge, A.; Zwijnenburg, M. A., Optical Excitation of MgO Nanoparticles; a Computational Perspective. Physical Chemistry Chemical Physics 2014, 16, 22052-22061. 
32. Peach, M. J. G.; Benfield, P.; Helgaker, T.; Tozer, D. J., Excitation Energies in Density Functional Theory: An Evaluation and a Diagnostic Test. The Journal of Chemical Physics 2008, $128,044118$.

33. Tozer, D. J.; Amos, R. D.; Handy, N. C.; Roos, B. O.; Serrano-Andres, L., Does Density Functional Theory Contribute to the Understanding of Excited States of Unsaturated Organic Compounds? Molecular Physics 1999, 97, 859-868.

34. Baerends, E. J.; Gritsenko, O. V.; van Meer, R., The Kohn-Sham Gap, the Fundamental Gap and the Optical Gap: The Physical Meaning of Occupied and Virtual Kohn-Sham Orbital Energies. Physical Chemistry Chemical Physics 2013, 15, 16408-16425.

35. Sushko, P. V.; Gavartin, J. L.; Shluger, A. L., Electronic Properties of Structural Defects at the MgO (001) Surface. The Journal of Physical Chemistry B 2002, 106, 2269-2276.

36. McKenna, K. P.; Shluger, A. L., First-Principles Calculations of Defects near a Grain Boundary in MgO. Physical Review B 2009, 79, 224116.

37. McKenna, K. P.; Shluger, A. L., Electron and Hole Trapping in Polycrystalline Metal Oxide Materials. Proceedings of the Royal Society A: Mathematical, Physical and Engineering Sciences 2011, 467, 2043-2053.

38. Hedin, L., New Method for Calculating the One-Particle Green's Function with Application to the Electron-Gas Problem. Physical Review 1965, 139, A796-A823.

39. Aryasetiawan, F.; Gunnarsson, O., The GW method. Reports on Progress in Physics 1998, 61, 237-312.

40. Golze, D.; Dvorak, M.; Rinke, P., The GW Compendium: A Practical Guide to Theoretical Photoemission Spectroscopy. Frontiers in Chemistry 2019, 7.

41. Salpeter, E. E.; Bethe, H. A., A Relativistic Equation for Bound-State Problems. Physical Review 1951, 84, 1232-1242.

42. Strinati, G., Application of the Green's Functions Method to the Study of the Optical Properties of Semiconductors. La Rivista del Nuovo Cimento (1978-1999) 1988, 11, 1-86.

43. Blase, X.; Duchemin, I.; Jacquemin, D.; Loos, P.-F., The Bethe-Salpeter Equation Formalism: From Physics to Chemistry. The Journal of Physical Chemistry Letters 2020, 11, 7371-7382.

44. Vosko, S. H.; Wilk, L.; Nusair, M., Accurate Spin-Dependent Electron Liquid Correlation Energies for Local Spin Density Calculations: A Critical Analysis. Canadian Journal of Physics 1980, 58, 1200-1211.

45. Lee, C.; Yang, W.; Parr, R. G., Development of the Colle-Salvetti Correlation-Energy Formula into a Functional of the Electron Density. Physical Review B 1988, 37, 785-789.

46. Becke, A. D., Density-Functional Thermochemistry. III. The Role of Exact Exchange. The Journal of Chemical Physics 1993, 98, 5648-5652.

47. Stephens, P. J.; Devlin, F. J.; Chabalowski, C. F.; Frisch, M. J., Ab Initio Calculation of Vibrational Absorption and Circular Dichroism Spectra Using Density Functional Force Fields. The Journal of Physical Chemistry 1994, 98, 11623-11627.

48. Grimme, S.; Antony, J.; Ehrlich, S.; Krieg, H., A Consistent and Accurate Ab Initio Parametrization of Density Functional Dispersion Correction (DFT-D) for the 94 Elements $\mathrm{H}$ Pu. The Journal of Chemical Physics 2010, 132, 154104.

49. Grimme, S.; Ehrlich, S.; Goerigk, L., Effect of the Damping Function in Dispersion Corrected Density Functional Theory. Journal of Computational Chemistry 2011, 32, 14561465. 
50. Weigend, F.; Ahlrichs, R., Balanced Basis Sets of Split Valence, Triple Zeta Valence and Quadruple Zeta Valence Quality for H to Rn: Design and Assessment of Accuracy. Physical Chemistry Chemical Physics 2005, 7, 3297-3305.

51. van Setten, M. J.; Weigend, F.; Evers, F., The GW-Method for Quantum Chemistry Applications: Theory and Implementation. Journal of Chemical Theory and Computation 2013, 9, 232-246.

52. Gui, X.; Holzer, C.; Klopper, W., Accuracy Assessment of GW Starting Points for Calculating Molecular Excitation Energies Using the Bethe-Salpeter Formalism. Journal of Chemical Theory and Computation 2018, 14, 2127-2136.

53. Holzer, C.; Klopper, W., Ionized, Electron-Attached, and Excited States of Molecular Systems with Spin-Orbit Coupling: Two-Component GW and Bethe-Salpeter Implementations. The Journal of Chemical Physics 2019, 150, 204116.

54. Perdew, J. P.; Burke, K.; Ernzerhof, M., Generalized Gradient Approximation Made Simple. Physical Review Letters 1996, 77, 3865-3868.

55. Krause, K.; Klopper, W., Implementation of the Bethe-Salpeter Equation in the Turbomole Program. Journal of Computational Chemistry 2017, 38, 383-388.

56. Martin, R. L., Natural Transition Orbitals. The Journal of Chemical Physics 2003, 118, 4775-4777.

57. Balasubramani, S. G., et al., Turbomole: Modular Program Suite for Ab Initio QuantumChemical and Condensed-Matter Simulations. The Journal of Chemical Physics 2020, 152, 184107.

58. Tasker, P. W., The Stability of Ionic Crystal Surfaces. Journal of Physics C: Solid State Physics 1979, 12, 4977-4984.

59. Cappellini, G.; Bouette-Russo, S.; Amadon, B.; Noguera, C.; Finocchi, F., Structural Properties and Quasiparticle Energies of Cubic SrO, $\mathrm{MgO}$ and $\mathrm{SrTiO}_{3}$. Journal of Physics: Condensed Matter 2000, 12, 3671-3688.

60. Schleife, A.; Fuchs, F.; Furthmüller, J.; Bechstedt, F., First-Principles Study of Groundand Excited-State Properties of MgO, ZnO, and CdO Polymorphs. Physical Review B 2006, 73, 245212.

61. Rinke, P.; Schleife, A.; Kioupakis, E.; Janotti, A.; Rödl, C.; Bechstedt, F.; Scheffler, M.; Van de Walle, C. G., First-Principles Optical Spectra for F Centers in MgO. Physical Review Letters 2012, 108, 126404.

62. Nejatipour, H.; Dadsetani, M., Excitonic Effects in the Optical Properties of Alkaline Earth Chalcogenides from First-Principles Calculations. Physica Scripta 2015, 90, 085802.

63. Gao, W.; Xia, W.; Gao, X.; Zhang, P., Speeding up GW Calculations to Meet the Challenge of Large Scale Quasiparticle Predictions. Scientific Reports 2016, 6, 36849.

64. Lambrecht, W. R. L.; Bhandari, C.; van Schilfgaarde, M., Lattice Polarization Effects on the Screened Coulomb Interaction W of the GW Approximation. Physical Review Materials 2017, 1, 043802.

65. Roessler, D. M.; Walker, W. C., Electronic Spectrum and Ultraviolet Optical Properties of Crystalline MgO. Physical Review 1967, 159, 733-738.

66. Schmidt-Grund, R.; Carstens, A.; Rheinländer, B.; Spemann, D.; Hochmut, H.; Zimmermann, G.; Lorenz, M.; Grundmann, M.; Herzinger, C. M.; Schubert, M., Refractive Indices and Band-Gap Properties of Rocksalt $\mathrm{Mg}_{\mathrm{x}} \mathrm{Zn}_{1-\mathrm{x}} \mathrm{O}(0.68 \leqslant \mathrm{X} \leqslant 1)$. Journal of Applied Physics 2006, 99, 123701. 
67. Begum, V.; Gruner, M. E.; Vorwerk, C.; Draxl, C.; Pentcheva, R., Theoretical Description of Optical and X-Ray Absorption Spectra of MgO Including Many-Body Effects. arXiv preprint arXiv:2012.08960 2020.

68. Whited, R. C.; Walker, W. C., Exciton Spectra of CaO and MgO. Physical Review Letters 1969, 22, 1428-1430.

69. Rao, A. S.; Kearney, R. J., Logarithmic Derivative Reflectance Spectra of BaO and SrO. physica status solidi (b) 1979, 95, 243-250.

70. Summers, G. P., Optical Absorption and Emission near the Band Edge of SrO. Physical Review B 1979, 20, 5275-5279.

71. Feldbach, E.; Kink, R.; Kirm, M.; Lushchik, A.; Lushchik, C.; Lôhmus, A.; Maaroos, A.; Martinson, I., Electronic Excitations and Uv Luminescence in SrO Crystals at $8 \mathrm{~K}$. Chemical physics letters 1995, 241, 597-602.

72. Ma, C.; Liu, T.; Chang, Q., Study on the Electronic Structures and Optical Absorption of F Center in the SrO Crystal with $\mathrm{G}_{0} \mathrm{~W}_{0}$-BSE. Computational and Theoretical Chemistry 2016, 1080, 79-83.

73. Riefer, A.; Fuchs, F.; Rödl, C.; Schleife, A.; Bechstedt, F.; Goldhahn, R., Interplay of Excitonic Effects and Van Hove Singularities in Optical Spectra: Cao and AIN Polymorphs. Physical Review B 2011, 84, 075218. 